\title{
A SOLUTION TO THE PROBLEM OF TIME-FRACTIONAL HEAT CONDUCTION IN A MULTI-LAYER SLAB
}

\author{
Urszula Siedlecka, Stanisław Kukla \\ Institute of Mathematics, Czestochowa University of Technology \\ Czestochowa, Poland \\ urszula.siedlecka@im.pcz.pl,stanislaw.kukla@im.pcz.pl
}

\begin{abstract}
In this paper a solution of the time-fractional heat conduction problem in a multilayer slab is presented. The boundary conditions of the third kind and the perfect contact at the interfaces are assumed. A space-time dependent volumetric heat source in the slab and time dependent surroundings temperatures are taken into account in the formulation of the problem. The solution is obtained in the form of a series expansion with respect to eigenfunctions of an auxiliary problem. A numerical example shows temperature distribution in the slab for various values of the order of the Caputo fractional derivative in the heat conduction equation.
\end{abstract}

Keywords: heat conduction, fractional derivative, multi-layer slab

\section{Introduction}

The heat conduction in layered slabs governed by a differential equation which is derived on the base of Fourier law have been considered by many authors [1-5]. An analytical form of exact solutions to such problems can be obtained in the case of heat conduction in a layered slab. Temperature distribution in multi-dimensional layered bodies in a rectangular coordinate system has been determined by HajiSheikh and Beck in paper [1]. The solution appointed by using the Green's function properties was utilized to numerical analysis of the temperature distribution in two-layer bodies. The temperature solution of the heat conduction problem for a multi-layer slab is presented by Özişik [2]. The solution in the form of a series of eigenfunctions was obtained.

Mathematical models based on generalizations of the Fourier law lead to fractional heat conduction. In these models the differential equation of the heat conduction includes the time- and/or space-fractional derivatives. The theory of the fractional calculus is contained in the books [3-5]. The methods to solutions of the fractional differential equations are presented in papers [6-9]. In Huang and Liu [6] to solve the Cauchy problem for the time-space fractional diffusion equation, temporal Laplace and spatial Fourier transforms have been applied. Demirci and 
Ozalp [7] use a transformation of the considered fractional differential equation in the equivalent fractional Volterra integral equation. Zheng and Wei [8] propose a regularization method to solving a Cauchy problem of the time-fractional diffusion equation. To solve the fractional heat equation, Anwar et al. [9] used a double Laplace transform.

The subject of this paper is the time-fractional heat conduction in a multilayer slab with boundary conditions of the third kind. The continuity of temperatures and the continuity of streams at the interfaces are assumed. The exact solution in the form of the series of eigenfunctions is obtained.

\section{Formulation of the problem}

Consider a slab consisting with $n$-layers which are characterised by thermal conductivity $\lambda_{i}$ and thermal diffusivity $a_{i}$. The heat conduction in the $i$-th layer is governed by the time-fractional differential equation

$$
\frac{\partial^{2} T_{i}}{\partial x^{2}}+\frac{1}{\lambda_{i}} q_{i}(x, t)=\frac{1}{a_{i}} \frac{\partial^{\alpha} T_{i}}{\partial t^{\alpha}}, \quad x \in\left[x_{i-1}, x_{i}\right], \quad 0<\alpha \leq 1, \quad i=1, \ldots, n
$$

where $T_{i}(x, t)$ and $q_{i}(x, t)$ is a temperature and a volumetric energy generation in the $i$-th layer, respectively, $x_{i-1}$ and $x_{i}$ are coordinates specifying surfaces of the $i$-th layer wherein $x_{0}=0, \alpha$ denotes an order of the Caputo fractional derivative with respect to time $t$. The Caputo derivative of order $\alpha$ is defined by [3]

$$
{ }_{0}^{C} D_{t}^{\alpha} f(t)=\frac{d^{\alpha} f(t)}{d t^{\alpha}}=\frac{1}{\Gamma(m-\alpha)} \int_{0}^{t}(t-\tau)^{m-\alpha-1} \frac{d^{m} f(\tau)}{d \tau^{m}} d \tau, \quad m-1<\alpha<m
$$

The functions $T_{1}$ and $T_{n}$ satisfy the boundary conditions

$$
\begin{gathered}
\lambda_{1} \frac{\partial T_{1}}{\partial x}\left(x_{0}, t\right)=-a_{L}\left(T_{L}(t)-T_{1}\left(x_{0}, t\right)\right) \\
-\lambda_{n} \frac{\partial T_{n}}{\partial x}\left(x_{n}, t\right)=-a_{R}\left(T_{R}(t)-T_{n}\left(x_{n}, t\right)\right)
\end{gathered}
$$

where $a_{L}, a_{R}$ are heat transfer coefficients and $T_{L}(t), T_{R}(t)$ are surrounding temperatures. Moreover, we assume that the temperatures $T_{i}(x, t)$ satisfy the conditions at the interfaces which correspond to the perfect contact of the layers

$$
T_{i}\left(x_{i}, t\right)=T_{i+1}\left(x_{i}, t\right), \quad i=1, \ldots, n-1
$$




$$
\lambda_{i} \frac{\partial T_{i}}{\partial x}\left(x_{i}, t\right)=\lambda_{i+1} \frac{\partial T_{i+1}}{\partial x}\left(x_{i}, t\right), \quad i=1, \ldots, n-1
$$

The temperature $T_{i}$ in the $i$-th layer for $t=0$ is given by

$$
T_{i}(x, 0)=f_{i}(x), \quad x \in\left[x_{i-1}, x_{i}\right], \quad i=1, \ldots, n
$$

The differential equation (1) and the conditions (3)-(7) constitute a complete formulation of the fractional heat conduction problem in the slab. A solution of the problem is presented in section 3 .

\section{Solution of the problem}

The solution to the problem (1)-(7) for temperature $T_{i}(x, t)$ in the $i$-th layer, we seek in the form of a sum

$$
T_{i}(x, t)=\theta_{i}(x, t)+\Phi_{i}^{1}(x) T_{L}(t)+\Phi_{i}^{2}(x) T_{R}(t), \quad i=1,2, \ldots n
$$

where the functions $\Phi_{i}^{1}, \Phi_{i}^{2}$ satisfy homogeneous differential equations and nonhomogeneous boundary conditions and the functions $\theta_{i}(x, t)$ satisfy nonhomogeneous fractional differential equations and homogeneous boundary conditions.

The differential equations and boundary conditions we obtain substituting (8) into equation (1) and (3)-(7). For the functions $\Phi_{i}^{p}, p=1,2$ we have

$$
\begin{gathered}
\frac{d^{2} \Phi_{i}^{p}(x)}{d x^{2}}=0, \quad i=1, \ldots, n, p=1,2 \\
\frac{\lambda_{1}}{a_{L}} \frac{d \Phi_{1}^{p}}{d x}(0)=\Phi_{1}^{p}(0)-\delta_{1, p} \\
\Phi_{i}^{p}\left(x_{i}\right)=\Phi_{i+1}^{p}\left(x_{i}\right), \quad i=1, \ldots, n-1, p=1,2 \\
\lambda_{i} \frac{d \Phi_{i}^{p}}{d x}\left(x_{i}\right)=\lambda_{i+1} \frac{d \Phi_{i+1}^{p}}{d x}\left(x_{i}\right), \quad i=1, \ldots, n-1, p=1,2 \\
-\frac{\lambda_{n}}{a_{R}} \frac{d \Phi_{n}^{p}}{d x}\left(x_{n}\right)=\Phi_{n}^{p}\left(x_{n}\right)-\delta_{2, p}
\end{gathered}
$$

where $\delta_{m, n}$ is Kronecker delta. The functions $\theta_{i}(r, t)$ satisfy the nonhomogeneous differential equation 


$$
\frac{\partial^{2} \theta_{i}(x, t)}{\partial x^{2}}+\frac{1}{\lambda_{i}} \tilde{q}_{i}(r, t)=\frac{1}{a_{i}} \frac{\partial^{\alpha} \theta_{i}(x, t)}{\partial t^{\alpha}}
$$

and the homogeneous conditions

$$
\begin{gathered}
\lambda_{1} \frac{\partial \theta_{1}}{\partial x}(0, t)=a_{L} \theta_{1}(0, t) \\
\theta_{i}\left(x_{i}, t\right)=\theta_{i+1}\left(x_{i}, t\right), \quad i=1, \ldots, n-1 \\
\lambda_{i} \frac{\partial \theta_{i}}{\partial x}\left(x_{i}, t\right)=\lambda_{i+1} \frac{\partial \theta_{i+1}}{\partial x}\left(x_{i}, t\right), \quad i=1, \ldots, n-1 \\
-\lambda_{n} \frac{\partial \theta_{n}}{\partial x}\left(x_{n}, t\right)=a_{R} \theta_{n}\left(x_{n}, t\right)
\end{gathered}
$$

The function $\tilde{q}_{i}$ in equation (14) is defined by

$$
\tilde{q}_{i}(x, t)=q_{i}(x, t)-\frac{\lambda_{i}}{a_{i}} \frac{d^{\alpha} T_{L}}{d t^{\alpha}} \Phi_{i}^{1}(x)-\frac{\lambda_{i}}{a_{i}} \frac{d^{\alpha} T_{R}}{d t^{\alpha}} \Phi_{i}^{2}(x)
$$

Moreover, by substituting equation (8) into equation (7), we obtain the initial condition in the form

$$
\theta_{i}(x, 0)=f_{i}(x)-\Phi_{i}^{1}(x) T_{L}(0)-\Phi_{i}^{2}(x) T_{R}(0)
$$

A solution of equation (9) is function

$$
\Phi_{i}^{p}(x)=A_{i}^{p}+B_{i}^{p} x, \quad p=1,2
$$

The coefficients $A_{i}^{p}$ and $B_{i}^{p}$ are determined by using conditions (10)-(13).

A solution of equation (14) we seek in the form of a series

$$
\theta_{i}(x, t)=\sum_{k=1}^{\infty} \Gamma_{k}(t) \Psi_{i, k}(x), \quad x_{i-1} \leq x \leq x_{i}, \quad i=1, \ldots, n
$$

where the function $\Psi_{i, k}(x)$, for fixed $i$ and $k$, is a solution of the following eigenproblem

$$
\begin{gathered}
\frac{d^{2} \Psi_{i, k}(x)}{d x^{2}}+\frac{\gamma_{k}^{2}}{a_{i}} \Psi_{i, k}(x)=0 \quad i=1, \ldots, n \\
\lambda_{1} \frac{d \Psi_{1, k}}{d x}(0)=a_{L} \Psi_{1, k}(0)
\end{gathered}
$$




$$
\begin{gathered}
\Psi_{i, k}\left(x_{i}\right)=\Psi_{i+1, k}\left(x_{i}\right), \quad i=1, \ldots, n-1 \\
\lambda_{i} \frac{d \Psi_{i, k}}{d x}\left(x_{i}\right)=\lambda_{i+1} \frac{d \Psi_{i+1, k}}{d x}\left(x_{i}\right), \quad i=1, \ldots, n-1 \\
-\lambda_{n} \frac{d \Psi_{n, k}}{d x}\left(x_{n}\right)=a_{R} \Psi_{n, k}\left(x_{n}\right)
\end{gathered}
$$

The general solution of equation (23) can be written in the form

$$
\Psi_{i, k}(x)=A_{i} \cos \beta_{i, k} x+B_{i} \sin \beta_{i, k} x
$$

where $\beta_{i, k}=\gamma_{k} / \sqrt{a_{i}}$. Substituting functions (28) into conditions (24)-(27) we obtain a homogeneous linear system of equations. The equation system in the matrix form reads

$$
\mathbf{D}_{k} \cdot \Psi_{k}=0
$$

where $\mathbf{D}_{k}=\left[d_{i j}^{k}\right]_{1 \leq i, j \leq 2 n}, \Psi_{k}=\left[\begin{array}{lllll}A_{1, k} & B_{1, k} & \ldots & A_{n, k} & B_{n, k}\end{array}\right]^{T}$. The elements of the matrix $\mathbf{D}_{k}$ are determined by using conditions (24)-(27). The non-zero solution of equation (29) exists when the condition

$$
\operatorname{det} \mathbf{D}_{k}=0
$$

is fulfilled. The equation (30) is solved numerically with respect to eigenvalues $\gamma_{k}$, $k=1,2, \ldots$. For the computed eigenvalues $\gamma_{k}$, the coefficients $A_{i}$ and $B_{i}$ occurring in equation (28) are determined by solving equation (29) with the assumption that $B_{n}=1$. The functions $\Psi_{i, k}$ satisfy the orthogonality condition

$$
\sum_{i=1}^{N} \frac{\lambda_{i}}{a_{i}} \int_{x_{i-1}}^{x_{i}} \Psi_{i, k}(x) \Psi_{i, k^{\prime}}(x) d x=\left\{\begin{array}{lll}
0 & \text { for } & k^{\prime} \neq k \\
N_{k} & \text { for } & k^{\prime}=k
\end{array}\right.
$$

where

$$
\begin{aligned}
& N_{k}=\frac{1}{4} \sum_{i=1}^{N} \frac{\lambda_{i}}{a_{i} \beta_{i, k}}\left[2 A_{i} B_{i}\left(1-\cos \left(2 \Delta x_{i, k}\right)\right)+\left(A_{i}^{2}-B_{i}^{2}\right) \sin \left(2 \Delta x_{i, k}\right)+2 \Delta x_{i, k}\left(A_{i}^{2}+B_{i}^{2}\right)\right] \\
& \text { and } \Delta x_{i, k}=\beta_{i, k}\left(x_{i}-x_{i-1}\right) .
\end{aligned}
$$

Substituting the function $\theta_{i}(x, t)$ in the form of the series (22) into equation (14) and using equation (23), after some transformation we obtain

$$
\sum_{k=1}^{\infty}\left[\frac{d^{\alpha} \Gamma_{k}}{d t^{\alpha}}+\gamma_{k}^{2} \Gamma_{k}(t)\right] \frac{\lambda_{i}}{a_{i}} \Psi_{i, k}(x)=\tilde{q}_{i}(x, t)
$$


Next, after multiplying the equation (32) by $\Psi_{i, k^{\prime}}(x)$, we integrate that equation successfully with respect to $x$ in the intervals $\left[x_{i-1}, x_{i}\right]$. In result, summing the equations for $i=1, \ldots, n$ and using the orthogonality condition (31), we obtain

$$
\frac{d^{\alpha} \Gamma_{k}}{d t^{\alpha}}+\gamma_{k}^{2} \Gamma_{k}(t)=\frac{1}{N_{k}} \sum_{i=1}^{n} \int_{x_{i-1}}^{x_{i}} \tilde{q}_{i}(x, t) \Psi_{i, k}(x) d x
$$

The differential equation (33) is complemented by an initial condition which we obtain using orthogonality condition (31) in equation (20). The initial condition assumes the form

$$
\Gamma_{k}(0)=\frac{1}{N_{k}} \sum_{k=1}^{n} \frac{\lambda_{i}}{a_{i}} \int_{x_{i-1}}^{x_{i}} \Psi_{i, k}(x) \tilde{f}_{i}(x) d x
$$

where $\tilde{f}_{i}(x)=f_{i}(x)-\Phi_{i}^{1}(x) T_{L}(0)-\Phi_{i}^{2}(x) T_{R}(0)$.

A solution of the problem (33)-(34) can be expressed by [11]

$$
\begin{aligned}
& \Gamma_{k}(t)=\frac{E_{\alpha}\left(-\beta_{k}^{2} t^{\alpha}\right)}{N_{k}} \sum_{i=1}^{n} \frac{\lambda_{i}}{a_{i}} \int_{x_{i-1}}^{x_{i}} \tilde{f}_{i}(x) \Psi_{i, k}(x) d x+ \\
& +\frac{1}{N_{k}} \int_{0}^{t}(t-\tau)^{\alpha-1} E_{\alpha, \alpha}\left(-\beta_{k}^{2}(t-\tau)^{\alpha}\right) \sum_{i=1}^{n} \int_{x_{i-1}}^{x_{i}} \tilde{q}_{i}(x, \tau) \Psi_{i, k}(x) d x d \tau
\end{aligned}
$$

where $E_{\alpha, \beta}$ is the Mittag-Leffler function [10] and $E_{\alpha}=E_{\alpha, 1}$.

Finally, the temperature distribution in the $i$-th sphere layer is given by equation (8) where the functions $\Gamma_{k}(t), \Psi_{i, k}(x), \Phi_{i}^{p}(x)$ are given by equations (35), (28) and (21), respectively.

\section{Numerical example}

The solution of the fractional heat conduction problem presented in section 3 can be used for the investigation of the effect of the parameters characterized the slab layers on the temperature distribution in the slab. In this section an illustrative example is given of a five-layer slab heated by an outer source which changes the ambient temperature periodically. Numerical computation were performed for a slab with the same thicknesses of the layers: $x_{i}-x_{i-1}=0.1 \mathrm{~m}(\mathrm{i}=1,2, \ldots, 5)$, and the following thermal diffusivities: $a_{1}=3.35 \times 10^{-6}, a_{2}=5.42 \times 10^{-6}$, $a_{3}=8.78 \times 10^{-6}, \quad a_{4}=1.42 \times 10^{-5}, \quad a_{5}=2.3 \times 10^{-5} \mathrm{~m}^{2} / \mathrm{s}^{\alpha}$, and thermal conductivi- 
ties: $\lambda_{1}=16.0, \lambda_{2}=24.0, \lambda_{3}=36.0, \lambda_{4}=54.0, \lambda_{5}=81.0 \mathrm{~W} /(\mathrm{m} \cdot \mathrm{K})$. The heat transfer coefficients were assumed as: $a_{L}=1200.0, a_{R}=400.0 \mathrm{~W} /\left(\mathrm{m}^{2} \cdot \mathrm{K}\right)$. The initial temperature $T_{0}$ and the ambient temperature $T_{R}$ were constants: $T_{0}=50 \mathrm{~K}, T_{R}=0$. The ambient temperature $T_{R}$ was a function of time: $T_{L}(t)=A+B \sin v t$, where $A=100, B=50 \mathrm{~K}$ and $v=2 \pi / 6000 \mathrm{~s}^{-1}$. Numerical calculations were carried out using the Mathematica package.

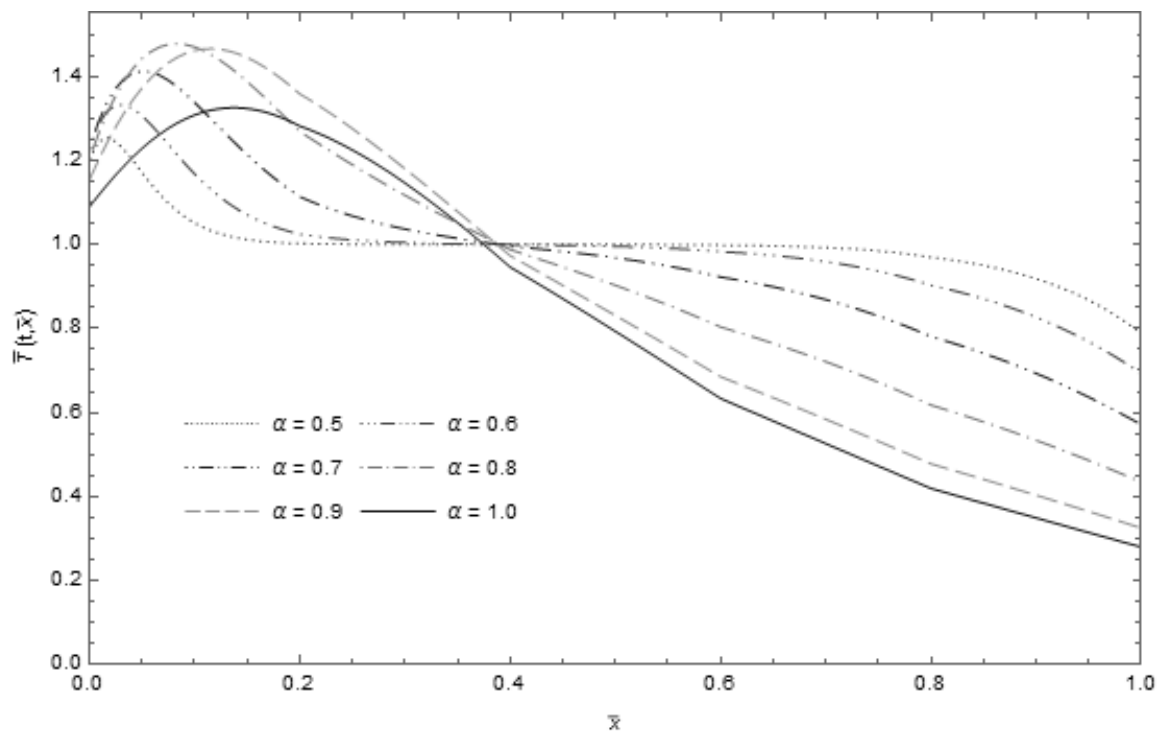

Fig. 1. Non-dimensional temperature $\bar{T}(t, \bar{x})=T(t, \bar{x}) / T_{0}$ as functions of $\bar{x}=x / d$ for $t=100 \mathrm{~d} / \sqrt{a_{n}}$ and different values of the derivative order $\alpha$

Non-dimensional temperatures: $\bar{T}(t, \bar{x})=T(t, \bar{x}) / T_{0}$, as functions of nondimensional distance from the left surface of the slab: $\bar{x}=x / d$, for $t=100 \mathrm{~d} / \sqrt{a_{n}}$ and $\alpha=0,5 ; 0,6 ; 0,7 ; 0,8 ; 0,9 ; 1,0$; are shown in Figure 1 . The lower temperatures on the right surface of the slab follow from this that the surroundings temperature is assumed as zero. Large differences of the temperatures in the slab can be observed for the heat conduction models with fractional derivative orders which are close to one, but for small orders these differences are slight.

\section{Conclusions}

The solution of the time-fractional heat conduction problem in a multilayer slab in the form of an eigenfunctions series is obtained. In the particular cases of functions characterized the volumetric heat source and surrounding temperatures, the 
integrals including the Mittag-Leffler function which occur in the solution can be determined in an analytical form. The particular sums of the series occurring in the problem solution can be numerically computed with desired accuracy by using the Mathematica package. Although the presented numerical example deals the fractional heat conduction in the five-layer slab, the solution can be used to determine the temperature distribution in a slab consisting of an arbitrary number of layers and particularly, to determine an approximate solution of this problem in a functionally graded slab.

\section{References}

[1] Haji-Sheikh A., Beck J.V., Temperature solution in multi-dimensional multi-layer bodies, International Journal of Heat and Mass Transfer 2002, 45, 1865-1877.

[2] Özişik M.N., Heat Conduction, Wiley, New York 1993.

[3] Podlubny I., Fractional Differential Equations, Academic Press, San Diego 1999.

[4] Klimek M., On Solutions of Linear Fractional Differential Equations of a Variational Type, The Publishing Office of Czestochowa University of Technology, Częstochowa 2009.

[5] Povstenko Y.Z., Fractional Thermoelasticity, Springer, New York 2014.

[6] Huang F., Liu F., The space-time fractional diffusion equation with Caputo derivatives, Journal of Applied Mathematics and Computing 2005, 19, 1-2, 179-190.

[7] Demirci E., Ozalp N., A method for solving differential equations of fractional order, Journal of Computational and Applied Mathematics 2012, 236, 2754-2762.

[8] Zheng G.H., Wei T., A new regularization method for a Cauchy problem of the time fractional diffusion equation, Advances in Computational Mathematics 2012, 36, 377-398.

[9] Anwar A.M.O., Jarad F., Baleanu D., Ayaz F., Fractional Caputo heat equation within the double Laplace transform, Romanian Journal of Physics 2013, 58, 1-2, 15-22.

[10] Mainardi F., Gorenflo R., On Mittag-Leffler-type functions in fractional evolution processes, Journal of Computational and Applied Mathematics 2000, 118, 283-299.

[11] Diethelm K., The Analysis of Fractional Differential Equations, Springer-Verlag, Berlin, Heidelberg 2010. 